\title{
La autonomía moral en el horizonte de la educación social
}

\section{Xus Martín}

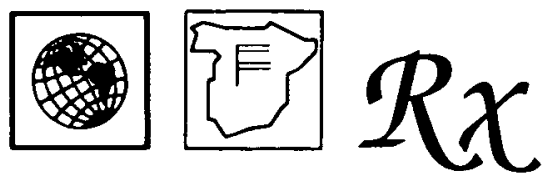

En este artículo se analiza algunas de las constantes más frecuentes en la personalidad del sujeto inadaptado y se propone un conjunto de metodologias que pueden ayudar en el tratamiento de las mismas. Así mismo se insiste en el ambiente institucional como elemento básico en el proceso de socialización y en el logro de la autonomía personal de cada individuo.

\section{LA EDUCACION SOCIAL COMO SOCIALIZACION}

Probablemente una de las ideas más compartidas entre un amplio sector de profesionales de la educación social es la convicción de que su intervención debe dirigirse a la socialización de los sujetos «inadaptados». El objetivo de esta intervención es ayudar al individuo a integrarse en su comunidad, dotándole de aquellas habilidades y del bagaje cultural necesario que le permita adaptarse al código establecido en la sociedad a la que pertenece. Se trata de que aquellas personas que durante mucho tiempo han permanecido al margen de la sociedad puedan integrarse y participar plenamente en ella, gozando, en la medida de lo posible, de las mismas oportunidades y derechos que el resto de los ciudadanos. Para que esto sea posible el sujeto «inadaptado» deberá adquirir e interiorzar de forma progresiva aquellas conductas, costumbres, valores, hábitos y normas existentes en su comunidad. Se pone el acento en la adaptación del individuo a la sociedad, entendiendo que sólo en la medida en que el sujeto muestre una conducta acorde con la mayoritaria, y su estilo de vida obedezca a los cánones establecidos, será aceptado y valorado socialmente. Pese a que se insiste en la capacidad del individuo para participar en el entramado social, la intervención educativa se dirige en gran parte a mostrar al sujeto de manera clara y contundente el «contenido social y cultural» que debe aprender. Es así como se le exigirá que adquiera hábitos convencionales (de higiene, de alimentación, de relaciones, etc), que incorpore en su vida los recursos y procedimientos socialmente valorados (por ejemplo, conseguir dinero mediante un trabajo), o que obedezca y respete las normas sociales (desde el código de circulación a la defensa de la propiedad). En definitiva, se trata de favorecer la adquisición progresiva de todo aquello que regula la vida en el exterior y que el individuo todavía no posee, ya sea por desconocimiento o por actitud de rebeldía ante la normativa social y el grupo mayoritario. Desde este punto de vista la tarea prioritaria del sujeto no consiste en detectar conflictos o en 
introducir modificaciones en el medio, ni tampoco en cuestionarse la corrección o no de las pautas culturales, ni de valorar en qué medida son justas o injustas las normas que rigen la sociedad, sino que deberá esforzarse por practicarlas, entenderlas, apreciarlas e interiorizarlas. La socialización supone, en este sentido, una cierta búsqueda de equilibrio y armonía entre individuo y medio social. El conflicto, si lo hay, queda en estos casos limitado a la dificultad para adaptarse y asumir el código vigente.

Desde un punto de vista más teórico uno de los grandes defensores de esta línea es $\mathrm{E}$. Durkheim, quien considera que el objetivo de la educación, también de la educación moral, es adaptar o ajustar a los individuos a la colectividad a la que pertenecen. Para ello establece una serie de elementos esenciales que deben ser tenidos en cuenta: el espíritu de disciplina, la adhesión a los grupos sociales y la autonomía de la voluntad (Durkheim, 1947). Según Durkheim una disciplina firme y estricta junto a una intensa vida colectiva permiten al sujeto reconocer la sociedad como valor superior a sí mismo, y le preparan para actuar en función de un interés colectivo y no meramente individual. Sólo a partir de estos dos primeros elementos el sujeto podrá conseguir la autonomía de la voluntad, por medio de la cual no sólo aceptará pasivamente las normas sociales sino que además conocerá la naturaleza de las mismas y comprenderá su necesidad.

Desde una línea más próxima al ámbito de la inadaptación social, la experiencia pedagógica llevada a cabo por Makarenko en la Colonia Máximo Gorki (Makarenko, 1977), obedece al ideal de la socialización y es bastante coherente con los elementos pedagógicos defendidos por Durkheim: disciplina firme y equilibrada junto a una intensa vida colectiva.

En esta línea podemos señalar, por ejemplo, las aportaciones que desde la psicología se han elaborado en torno a la modificación de conducta. Durante muchos años, y todavía en la actualidad, se han generado proyectos educativos muy centrados en el cambio comportamental y en el aprendizaje y adopción de hábitos y conductas convencionales. Los programas de habilidades sociales que se están llevando a cabo en distinas instituciones penitenciarias de nuestro país, así como en proyectos educativos con adolescentes, trabajan fundamentalmente en esta línea.

Evidentemente ha habido otras aportaciones en el ámbito de la educación social cuyo objetivo no era la socialización. En algunos casos incluso se llegó a afirmar que no se debía adaptar a los sujetos inadaptados a la sociedad sino prepararlos para que llevaran a cabo el cambio social (Deligny, 1971). Sin embargo, éstas han sido posturas minoritarias y en todo caso menos compartidas en la intervención real con población inadaptada.

El ideal socializador y la manera de entender la educación vinculada a mecanismos de adaptación heterónoma a las normas sociales plantea algunos interrogantes y limitaciones. En concreto, no parece estar nada claro qué protagonismo tiene el sujeto que se «socializa», ni como se entiende una autonomía "más autónoma» que la que Durkheim le concede. Desde estas posturas la responsabilidad del sujeto en su proceso de formación moral es muy limitada, ya que no consiste en construir formas nuevas de vida, sino en hacer suyas las influencias impuestas desde fuera. $Y$ es en torno a las limitaciones e interrogantes que plantea la postura eminentemente socializadora, en donde se sitúa la aportación de la educación moral tal y como nosotros la entendemos: una educación orientada hacia la construcción de la autonomía.

\section{LA EDUCACION SOCIAL COMO CONSTRUCCION DE LA AUTONOMIA}

Junto a la necesidad de que el individuo se adapte a la sociedad en la que vive, hay otros aspectos, no contradictorios con el proceso de socialización, que consideramos también deben contemplarse desde la intervención educativa. En este sentido, la educación para la autonomía recoge e incorpora los mecanismos de adquisición e 
interiorización de las pautas sociales, pero a su vez insiste en la creación de personalidades autónomas. La construcción de la autonomía aporta al sujeto aquellas herramientas o instrumentos que le permiten incorporarse de forma positiva a la colectividad, a la sociedad a la que pertenece, pero que también le permiten un importante nivel de autonomía para tomar decisiones que configuran su forma de ser y su manera de vivir.

En el ámbito de la intervención con sujetos inadaptados creemos que esto es especialmente significativo, dado que en muchos casos los problemas con los que nos encontramos están estrechamente vinculados con carencias en el propio desarrollo integral del sujeto que impiden la autonomía personal. Carencias que aunque la mayoría de las veces han sido provocadas por un medio hostil al individuo y por procesos de socialización con graves alteraciones, han quedado cristalizadas en la personalidad del sujeto inadaptado.

A continuación vamos a describir algunas de estas carencias que con mayor frecuencia se perciben en la personalidad de los sujetos inadaptados. Antes de presentarlas, deberíamos señalar el riesgo y el error que siempre lleva implícita la descripción de un "perfil» de personalidad y la generalización al hablar de personas. Es obvio que cualquiera de las carencias o limitaciones que a continuación señalamos pueden encontrarse en sujetos considerados habitualmente como «adaptados» a la sociedad, de la misma manera que algunas de ellas no se presentan en personas consideradas habitualmente como «sujetos inadaptados». Sin embargo, la descripción de este perfil hipotético de sujeto inadaptado nos puede ayudar a definir el tipo de estrategias más adecuadas y oportunas en el ámbito de la inadaptación social.

\section{Carencias en la personalidad del sujeto inadaptado}

- La impulsividad. Consiste en actuar de forma precipitada y sin pensar sobre la acción que se va a llevar a cabo. Las personas impulsivas actúan en función de sus deseos o ideas momentaneas, sin valorar las distintas alternativas ni las consecuencias que de ellas se derivan. Si bien a nivel externo lo que se aprecia es una conducta precipitada e impulsiva, a nivel interno muchas de estas personas carecen de las destrezas cognitivas necesarias para planificarse a corto o medio plazo.

- Escaso sentimiento de culpabilidad. Los sujetos con tendencia a la externalidad consideran que aquello que ocurre en sus vidas no depende de ellos mismos sino de personas, circunstancias y acontecimientos externos. El azar, el destino o la suerte suelen ser los elementos que determinan lo que ocurre en cada momento y la persona se encuentra indefensa y sin poder para controlar su vida. Todo ello tiene como consecuencia ( o como causa?) que la persona no se sienta responsable de sus actos y por lo tanto tampoco se considere culpable de aquellas conductas que han originado un mal a otros.

- Carencia de intimidad. En distintas investigaciones se ha puesto de manifiesto el «vacío» que de sí mismo tiene el sujeto inadaptado. Es frecuente que éste ni siquiera tenga una clara visión de los datos más relevantes de su propia biografía (sucesión de los acontecimientos que han ocurrido en su vida). Asimismo son personas con dificultades para asumir su historia personal, personas que mantienen pocas y débiles vinculaciones con el pasado, que viven sin enraizarse en el presente y que sobre todo no proyectan hacia el futuro.

- Pensamiento concreto. La dificultad de algunos sujetos para pensar en algo que no se hace patente a su percepción inmediata, les complica notablemente la comprensión del mundo. Cuando una persona carece de pensamiento abstracto está en desventaja social en relación al resto de individuos, ya que no tiene las mismas posibilidades para entender ideas, puntos de vista (y también sentimientos) distintos a los propios. 
- Rigidez de pensamiento. Cuando el pensamiento es inflexible, rígido y permanece cerrado a nuevas ideas y puntos de vista, los sujetos suelen mostrarse intolerantes y dogmáticos en sus relaciones con los demás. Con frecuencia este tipo de pensamiento se traduce en comportamientos agresivos e impositivos que perturban de manera considerable la convivencia en grupo.

- Adaptabilidad situacional. La necesidad de buscar prestigio y reconocimiento junto con el clima de desconcierto en el que han crecido gran parte de los sujetos «inadaptados» les dota de una gran adaptabilidad a situaciones diversas e incluso opuestas. La falta de criterios claros y puntos de referencia nítidos les permite "normalizar" situaciones nuevas y conflictivas, así como reaccionar rápidamente llevando a cabo comportamientos contradictorios.

- Dificultad para la resolución de problemas. Esta dificultad se concreta en cuatro aspectos básicos. Uno, la dificultad para reconocer una situación conflictiva como tal; dos, la incapacidad para considerar situaciones alternativas al problema; tres, la imposibilidad para calcular las consecuencias de su comportamiento; y cuatro, la no comprensión de las relaciones causa-efecto entre la propia conducta y la reacción de los demás.

- Egocentrismo. La persona egocéntrica sólo puede apreciar, entender y plantearse el mundo y cuanto en él ocurre desde su propia perspectiva. No sólo no entiende los puntos de vista y sentimientos ajenos sino que además tampoco puede calcular el efecto que su conducta o actitud tendrá en el otro. Ello le dificulta establecer relaciones interpersonales basadas en la comprensión y el mutuo entendimiento. Este déficit ha sido uno de los más estudiados en relación a la conducta delictiva y en concreto a la falta de conciencia que muchos delincuentes tienen sobre las consecuencias que su comportamiento ha tenido para la víctima.

Si tal y como afirman distintos autores (Ross, 1992; Valverde, J., 1988) estas carencias o limitaciones son relativamente comunes entre la población inadaptada, entendemos que la intervención educativa debe orientarse al trabajo sistemático de estos déficits o carencias.

Es así como frente a la impulsividad deberá favorecerse el ejercicio de la reflexión, el análisis y la consideración de las distintas alternativas o respuestas posibles ante una situación determinada. El escaso sentimiento de culpabilidad y de responsabilidad debe dar paso de forma progresiva a una actitud responsable y comprometida con el medio y con las demás personas. La carencia de intimidad y el vacío de sí mismo que experimenta el sujeto inadaptado tiene que afrontarse desde un proceso de autoconocimiento y autoaceptación de la propia historia y la propia persona. El pensamiento concreto que caracteriza la comprensión que se tiene del mundo deberá evolucionar hacia un pensamiento abstracto, capaz de entender también aquello que no se percibe de forma inmediata y que no llega a través de los sentidos. Asimismo el dogmatismo y la rigidez deberán avanzar hacia una mayor tolerancia y flexibilidad, que permita la apertura a ideas nuevas y puntos de vista diversos. La adaptabilidad situacional deberá debilitarse en beneficio de una formación de la persona más estable y centrada, con puntos de referencia y criterios de actuación que no sólo dependen de las circunstancias sino también del sujeto. Por último, el egocentrismo que está en la base del resto de limitaciones deberá avanzar hacia un progresivo descentramiento en el que de forma paulatina el sujeto desarrolle y consolide su perspectiva social.

\section{METODOLOGIAS PROPIAS DE LA EDUCACION PARA LA CONSTRUCCION DE-LA AUTONOMIA}

Parece ser que es hacia la consecución de estas modificaciones en la personalidad del sujeto inadaptado hacia donde, en un principio, debería encaminarse la intervención. Desde el ámbito de la educación para la autonomía se propone una serie de metodologías y técnicas cuyo objetivo es desarrollar y construir las distintas 
capacidades y dimensiones que están implícitas y definen la personalidad moral. En unos casos se apuntará más hacia los procesos de socialización y en otros hacia la construcción de la autonomía personal. Sin embargo, en la realidad inmediata la línea divisoria entre ambos aspectos quizás no sea siempre tan nítida como aparece en el discurso teórico. Pasamos a continuación a señalar las metodologías más representativas indicando sus principales aportaciones. (Puig, 1995).

\begin{tabular}{|c|c|c|}
\hline CARENCIAS & CAPACIDADES & METODOLOGÍAS \\
\hline Impulsividad & Reflexividad & Autorregulación \\
\hline Escaso sentido de culpa & Responsabilidad & Enfoques socioafectivos \\
\hline Carencia de intimidad & Autoconocimiento & Escritura autobiográfica \\
\hline Pensamiento concreto & Pensamiento abstracto & Discusión dilemas morales \\
\hline Rigidez de pensamiento & Tolerancia & Comprensión crítica \\
\hline $\begin{array}{l}\text { Dificultad en la resolución } \\
\text { de problemas }\end{array}$ & Juicio moral & $\begin{array}{l}\text { Estrategias de resolución } \\
\text { de conflictos }\end{array}$ \\
\hline Egocentrismo & Descentración & $\begin{array}{l}\text { Role-Playing } \\
\text { Clarificación de valores } \\
\text { Habilidades sociales }\end{array}$ \\
\hline
\end{tabular}

\section{Clarificación de valores}

La metodología de clarificación de valores intenta ofrecer a los educadores una serie de recursos para ayudar a los sujetos a ejercitarse en el proceso de valoración, descubriendo y tomando conciencia de aquellos aspectos que son importantes en su vida. Este método no se basa ni tiene como finalidad «enseñar valores», sino facilitar la construcción o adopción de valores personales. Ello es especialmente significativo en aquellos casos en que el sujeto está sometido a modelos, propuestas y exigencias sociales con frecuencia contradictorias.

Este método parte de una idea de "valor» íntimamente ligada a la idea de "proceso», en el cual lo fundamental no son los valores en sí mismos sino la capacidad del sujeto para adquirir valores que le faciliten un mayor autoconocimiento y unas relaciones positivas y satisfactorias con los demás. Este proceso de valoración consta de tres fases: la selección del valor, la apreciación de los valores elegidos, y la actuación acorde con dichos valores.

\section{Escritura autobiográfica}

Veíamos anteriormente como una de las constantes que aparece de forma reperida en los sujetos inadaptados es la dificultad para asumir la propia historia. Los ejercicios autobiográficos se orientan de manera privilegiada a tratar esta limitación favoreciendo en la persona la elaboración de su identidad personal en tanto que historia de la propia experiencia vital. Se intenta que cada individuo reconozca, asuma y dé sentido a su pasado y que progresivamente construya, imagine y proyecte de alguna manera el futuro que desea. Ambos aspectos, entender el propio pasado y proyectar el futuro, son condiciones fundamentales que deberán trabajarse de forma paralela. 


\section{Discusión de dilemas morales}

La discusión de dilemas morales tiene como objetivo prioritario el desarrollo del juicio moral. Esta metodología considera que las situaciones moralmente controvertidas son las que más estimulan el desarrollo de las formas de pensamiento sobre temas morales. Y ello porque la percepción de un conflicto moral crea en el sujeto una situación de crisis en la que se rompe la seguridad y confianza en sus propios criterios, lo cual le obliga a buscar nuevas razones que le permitan solucionar el dilema y que le devuelvan la seguridad en sus propios criterios morales. Esta metodología permite trabajar en torno a algunas de las carencias anteriormente señaladas: rigidez de pensamiento, pensamiento concreto, la dificultad para resolver problemas y en general todas aquellas limitaciones que hacen referencia a la dimensión más cognitiva de la persona.

\section{Role-playing}

El desarrollo de la perspectiva social y la empatía debería ser uno de los objetivos prioritarios en la intervención con población inadaptada, dado que el egocentrismo es una de sus mayores limitaciones. Trabajar la asunción de roles supone contemplar la adquisición de la habilidad para comprender que los otros tienen también sentimientos y puntos de vista sobre los sucesos, la habilidad para relacionar distintos elementos, y la habilidad para relativizar la propia opinión cuando se trate de juzgar el punto de vista de los demás.

Los ejercicios de role-playing, pretenden facilitar el desarrollo de la perspectiva social y en ellos se trabaja conjuntamente los aspectos interpersonales, racionales y emocionales que actúan en la toma de decisiones. A partir de ellos, los individuos exploran los sentimientos, actitudes, valores, razones y percepciones que influyen en su conducta, a la vez que se sienten forzados a descubrir y aceptar el rol de los demás.

\section{Comprensión crítica}

A diferencia de los dilemas morales, la comprensión crítica intenta facilitar básicamente la contextualización del juicio moral proporcionando una situación compleja y rica en información. Se parte del amplio marco referencial de cada sujeto (emociones, sentimientos, intuiciones, conocimientos, y prejuicios personales) que le permite proceder a una interpretación global de la realidad. La posición de cada persona es un elemento clave que debe ser reconocido por el grupo. Sin embargo, reconocer y acoger los diferentes puntos de partida no niega la posibilidad de llegar a un consenso respecto a un problema determinado. La finalidad de estos métodos es precisamente impulsar el entendimiento entre las "diferentes voces" que se originan en torno a un conflicto, entendimiento que implica una actitud de diálogo y de interés hacia los motivos que tiene cada una de las personas para pensar de una determinada manera. Se trata, pues, de entrar en diálogo con los demás a la vez que se entra en diálogo con uno mismo.

\section{Enfoques socioafectivos}

Los ejercicios socioafectivos tienen como objetivo el desarrollo de la sensibilidad para reconocer situaciones conflictivas y para sentirse afectado por ellas y se centran de forma primordial en el trabajo de las emociones y los sentimientos, entendiendo que éstos deben derivar hacia reflexiones a propósito de los mismos, así como a la adopción de actitudes acordes con ellos. Si una de las características del sujeto inadaptado era su escaso sentimiento de culpabilidad ante las consecuencias negativas que su conducta genera a un tercero (la víctima), desarrollar la sensibilidad de «sentirse afectado" debe ser una de las finalidades claves de la intervención educativa. 
Estrategias para la resolución de conflictos

Las actividades que se proponen desde el método de la resolución de conflictos pretenden contribuir al aprendizaje de habilidades concretas que faciliten al sujeto la resolución satisfactoria de los conflictos. Para ello se llevan a cabo ejercicios de reconocimiento del problema, de identificación del mismo y de conceptualización del conflicto. Asimismo se realizan actividades destinadas a idear distintas alternativas en torno al conflicto así como a imaginar las consecuencias que se deriva de cada una de las soluciones propuestas. Junto a la adquisición de habilidads concretas, estas metodologías intentan favorecer en el educando una actitud constructiva y respetuosa en su relación con los demás.

\section{Habilidades sociales}

Los ejercicios de habilidades sociales tienen como objetivo la adquisición de normas convivenciales que permitan una correcta vida colectiva. Se trata de favorecer e intensificar procesos de socialización del sujeto, socialización entendida como adaptación a los usos sociales vigentes. Junto a los contenidos convencionales concretos que se desean transmitir, también se insiste en la adopción e interiorización progresiva de la conducta asertiva, en detrimento de actitudes agresivas o pasivas que pueda tener el sujeto. Estas metodologías no tratan tanto la autonomía personal como los aspectos heterónomos que son valorados positivamente por el medio sociocultural y que el sujeto todavía no posee. Podemos decir que mediante la práctica de estos ejercicios se están trabajando todas aquellas carencias que el individuo tiene en sus relaciones interpersonales y, sobre todo, en sus relaciones con el grupo socialmente mayoritario.

\section{Metodologías basadas en la autorregulación}

Las metodologías basadas en la autorregulación se centran de forma muy prioritaria, aunque no exclusiva, en la vertiente más comportamental y tienen como objetivo desarrollar las capacidades de dirección de sí mismo de cada sujeto. Pretenden ayudarle a conseguir un elevado grado de coherencia entre su juicio y la acción que realiza, facilitándole la formación de hábitos conductuales acordes con sus criterios personales. No se trata sin embargo de omitir, ignorar o eliminar las adquisiciones sociales y culturales de cada sujeto, sino de dotarle de aquellos criterios y capacidades que le permitan tomar el control de su propia vida. Este tipo de metodologías son un intento por poner en las manos del individuo la responsabilidad de su historia e implicarle en la construcción de su futuro inmediato, evitando o disminuyendo la tendencia a creer que cuanto ocurre en su vida depende de factores externos sobre los cuales él no tiene ningún poder.

\section{LA SIGNIFICATIVIDAD DE LOS CONTENIDOS Y EL PAPEL DE LA INSTITUCION EN LA PERSONALIDAD DEL SUJETO INADAPTADO}

\section{Las experiencias de vida como contenido}

Una vez señaladas las distintas metodologías que desde la educación como construcción de la autonomía se proponen, es necesario señalar asimismo que éstas sólo podrán ser útiles en el ámbito de la inadaptación social cuando se apliquen a propósito de contenidos significativos para los sujetos con los que se trabaja. Es decir, cuando los dilemas morales que se discutan supongan realmente un conflicto 
de valor para el grupo al que nos dirigimos, o cuando los ejercicios de role-playing o de comprensión crítica recojan situaciones cercanas a la experiencia de los usuarios. Creemos que los contenidos, o temas sobre los que se habla, se discute, se reflexiona y se diseñan conductas en el ámbito de la inadaptación es un elemento clave en la intervención, del cual no se puede prescindir. Las metodologías o estrategias propuestas sólo tendran sentido en la medida en que cuestionen a los sujetos sobre sus propias vidas y en la medida en que éstos perciban una relación estrecha entre los contenidos que se tratan y su realidad cercana. En este sentido pensamos que sería conveniente realizar diversos estudios e investigaciones que nos ayudaran a conocer qué hechos, situaciones y experiencias son percibidos como moralmente significativos para los sujetos de distintos proyectos educativos de integración social.

\section{El papel de las instituciones educativas en el desarrollo personal del sujeto inadaptado}

Hemos centrado este artículo en las metodologías propias de la educación moral que nos permiten trabajar las carencias detectadas en el desarrollo personal del sujeto inadaptado. Sin embargo, y pese a la importancia de este elemento, debemos considerar otro aspecto que creemos imprescindible para llevar a cabo una intervención educativa correcta y eficaz. Nos estamos refiriendo al papel que tienen las instituciones educativas en el desarrollo y la formación de la personalidad de los sujetos que allí se educan. Si bien no es nuestra intención desarrollar ampliamente el tema, sí que deseamos dedicar unas líneas a subrayar su importancia en el ámbito de la educació social.

Uno de los elementos que de manera ímplicita o explícita, voluntaria o involuntaria tienen mayor repercusión en la formación de la personalidad moral de los sujetos inadaptados es la institución o instituciones educativas a las que pertenecen. Es decir, las personas aprenden actitudes y valores por el simple motivo de crecer en una institución que tiene unas determinadas formas de organización, unas estructuras de poder, unos canales de participación, unas normativas y reglamentos instituidos, en definitiva, unas relaciones sociales que la definen. Obviamente las maneras en que todos estos aspectos se especifiquen y desarrollen determinará y generará procesos educativos distintos. En este sentido podemos afirmar que cada organización institucional expresa determinadas opciones y principios morales a la vez que impone a sus miembros climas morales concretos.

Las relaciones interpersonales que se establecen en los distintos proyectos educativos con sujetos inadaptados son probablemente una de las principales fuentes de aprendizaje sociomoral. Estas no son nunca sustituídas ni quedan suplidas por otro tipo de intervención. A pesar de la importancia que tienen las actividades específicas de educación moral en el desarrollo moral del individuo, las relaciones personales que establece con sus compañeros y educadores favorecen o dificultan la formación de una personalidad autónoma y solidaria. Es por tanto tarea de las instituciones educativas favorecer relaciones personales que propicien personalidades maduras; es decir, relaciones basadas en la libertad y la cooperación entre sus diferentes miembros. La vivencia de experiencias de autonomía, diálogo y cooperación deberá ser uno de los objetivos básicos en los proyectos de educación social, que en función de la edad de los sujetos se llevará a cabo de distintas maneras. Los miembros del grupo, ya en edades tempranas, deberan ejercer su derecho a la participación, acostumbrándose a dialogar, discutir puntos de vista distintos, respetar a aquellos que opinan diferente, $e$ intentar llegar a acuerdos y consensos respecto a los temas que afectan al colectivo. La discusión respecto a los conflictos cotidianos de la vida del grupo, la adopción de normas, la crítica constructiva de aquellos aspectos que no comparten y la aceptación de las decisiones colectivas son todos ellos elementos que irán desarrollando en los niños y niñas las capacidades descritas anteriormente. Destacamos en 
relación con todo ello la práctica sistemática de la asamblea de grupo, como uno de los métodos más emblemáticos en aquellos proyectos educativos orientados a la participación activa de sus miembros.

La participación democrática en la institución educativa potenciará, por ejemplo, el desarrollo del juicio y acción moral, ayudando a los individuos a superar el egocentrismo que les impide comprender puntos de vista ajenos y les dificulta la asimilación personal de las normas morales. Ello requiere limitar las relaciones interpersonales que se basan únicamente en el respeto unilateral, y potenciar un tipo de relaciones en las que la imposición autoritaria es sustituida por la cooperación y el respeto mutuo entre iguales ${ }^{1}$.

Llevar a cabo una experiencia como la que estamos describiendo, es decir, una experiencia de participación democrática en el ámbito educativo, es prácticamente imposible si no se dan dos condiciones básicas. En primer lugar, se hace necesaria la generalización del diálogo entre todos los participantes. En este sentido el educador deberá propiciar el intercambio de opiniones como ejercicio común en la dinámica del colectivo. Esto significa que además de institucionalizar la asamblea como momento privilegiado de gestión colectiva, se estará atento a generar diálogo e intercambio en cualquier situación y acontecimiento que tenga lugar en el grupo. En segundo lugar, es imprescindible que la actitud del educador sea coherente con la propuesta que aquí defendemos. Probablemente una actitud excesivamente directiva y autoritaria no favorezca el diálogo ni la gestión autónoma de la vida colectiva. Por el contrario, una actitud respetuosa, activa, paciente y firme, ayudará a los educandos no sólo a encontrar soluciones a los problemas concretos sino que favorecerá en éstos la adquisición del diálogo como principal instrumento organizativo.

Entendemos, pues, que los elementos señalados en este artículo: metodologías, contenidos significativos, participación en la institución y actitud de los educadores son todos ellos complementarios e imprescindibles para una adecuada intervención en el ámbito de la educación social. Creemos que no partimos de cero y que en distintos proyectos hay educadores y educadoras trabajando en esta línea.

\section{Notas}

(1) En el ámbito de la intervención con sujetos inadaptados ha habido distintos proyectos basados en el sistema de autogobierno. Entre ellos citamos, una vez más, la experiencia de $A$. S. Makarenko en la Colonia Maximo Gorki, dirigida a adolescentes predelincuentes y en las que los usuarios tenían un altísimo nivel de participación en el funcionamiento y gestión de su comunidad.

\section{Referencias}

AA.VV. (1991). Pedagogia de la marginación. Madrid: Popular, col. Trabajo social-Política social.

COSTA, M. y LOPEZ, E. (1991). Manual para el educador social, vol. 1 y 2. Madrid: Ministerio de Asuntos Sociales.

DEUGNY, F. (1971). Los vagabundos eficaces. Barcelona: Estela.

DURKHeim, E. (1947). La educación moral. Buenos Aires: Losada.

Ross, R. (1987). «Razonamiento y Rehabilitación: un programa cognitivo para el tratamiento y la prevención de la delincuencia». En Garrido, V. y Montoro, L. (coords.): Lecturas de pedagogía correccional. Valencia: Nau llibres.

Makarenko, A.S. (1977). Poema pedagógico. Barcelona: Planeta, col. Popular.

PUIG, J. M. (1995). La educación moral en la enseñanza obligatoria. Barcelona: Horsori-ICE, col. Cuadernos de Educación, 17.

RutTer, M. y GrLer, H. (1983). Delincuencia juvenil. Barcelona: Martínez Roca, col. Biblioteca de Psicología, Psiquiatría y Salud.

VAlVerde Molina, J. (1988): El proceso de inadaptación sacial. Madrid: Popular.

VEGA, A. (1991). Pedagogía de los inadaptados sociales. Madrid: Narcea. 


\section{La autonomía moral en el horizonte} de la educación social Xus Martín CL\&E, 1995, 27, pp. 21-30

Resumen: Este artículo intenta aportar algunas sugerencias que puedan ser útiles en la intervención educativa con población inadaptada. La primera parte del artículo es una reflexión en torno a las finalidades u objetivos que desde la educación social se deben perseguir. A continuación se hace una aproximación al sujeto inadaptado señalando las carencias que con frecuencia se detectan en su personalidad y se propone metodologías concretas que permitan rrabajar rales carencias o limitaciones. En el último apartado del artículo se señala la importancia que los contenidos y el clima de la institución tienen en el proceso evolutivo de estos sujetos.

Datos sobre la autora: Xus Martín es Profesora Titular de Educación Moral en la Facultad de Pedagogía de la Universidad de Barcelona y miembro del Grupo de Investigación en Educación Moral (GREM) del Departamento de Teoría e Historia de la Educación de la U.B. Anteriormente había trabajado como educadora en proyectos educativos con adolescentes inadaptadas.

Dirección: Departamento de Teoría e Historia de la Educación, Facultad de Pedagogía, Universidad de Barcelona, Baldiri Reixac, s/n 08028 Barcelona. Tel (93) 4409200.

(C) PERMISOS PARA CITAR O REPRODUCIR EN OTRAS FUENTES: Se pueden citar libremente hasta 500 palabras. Para reproducir una porción de texto mayor, figuras o ilustraciones, se deberá pedir permiso por escrito a la revista, especificando el uso al que se destina el texto. En todos los casos, se deberá citar el copyright de $C L \& E$. En el caso de artículos o textos que hayan sido a su vez reproducidos en $C L \& E$ los interesados deberán dirigirse tanto a los detentadores del copyright original como a $C L \& E$, en el caso de que se quiera hacer uso de la traducción. FO'TOCOPIAS: Para todo lo relacionado con el uso mediante fotocopia del material de esta revista, deberán dirigirse a: CEDRO, C/ José Marañón, 10, $3^{\circ}$ Izda. Tel. 5941575 . Fax 4453567 